\title{
Hemodynamic Effects of Developmental Venous Anomalies with and without Cavernous Malformations
}

\author{
A. Sharma, G.J. Zipfel, C. Hildebolt, and C.P. Derdeyn
}

\begin{abstract}
BACKGROUND AND PURPOSE: Association between developmental venous anomalies is well known, but remains unexplained. Our aim was to study possible hemodynamic differences around developmental venous anomalies with and without cavernous malformations.

MATERIALS AND METHODS: In this prospective study approved by the institutional review board, PWI was performed in 24 patients with 25 DVAs (10 with and 15 without CMs) who consented to participate. We calculated relative cerebral blood volume, relative cerebral blood flow, and relative mean transit time for the brain surrounding the DVA tributaries in reference to contralateral mirror image locations. Corresponding control values (CCBV, CCBF, and CMTT) were generated in a similar fashion for remote ipsilateral regions with normal venous drainage, also in reference to contralateral mirror image locations. Perfusion parameters for DVAs and control regions were tested for differences between groups with the $t$ test for independent or paired samples (or the nonparametric equivalents). Similar testing was done for perfusion parameters for DVAs with and without CMs.

RESULTS: Normal-appearing brain surrounding DVAs showed increased rCBV (median $=2.98$; range $=1.39-6.61$ ), increased rCBF (median $=2.00$, range $=0.79-4.43$ ), and increased rMTT (mean $=1.46 ; 95 \%$ confidence interval, 1.32-1.59). These were significantly higher than median CCBV (0.99; 95\% confidence interval, 0.89-1.06; $P<.01)$, median cCBF (1.00; 95\% confidence interval, 0.94-1.27; $P<.01)$, and mean CMTT (1.00; 95\% confidence interval, 0.98-1.02; $P<.01)$, respectively. Mean rMTT (1.70; 95\% confidence interval, 1.46-1.93) for DVAs with CMs was higher than mean rMTT (1.29; 95\% confidence interval, 1.19-1.40; $P<.01)$ for DVAs without CMs.
\end{abstract}

CONCLUSIONS: DVAs are strongly associated with altered hemodynamics. Significant differences in these hemodynamic alterations for DVAs with and without CMs suggest their possible role in the formation of CMs.

ABBREVIATIONS: $c C B F=$ relative $C B F$ for control brain parenchyma with normal venous drainage; $c C B V=$ relative $C B V$ for control brain parenchyma with normal venous drainage; $C M$ = cavernous malformation; $C M T T$ = relative MTT for control brain parenchyma with normal venous drainage; DVA = developmental venous anomaly; $r$ = relative (for CBV, CVF, MTT)

D evelopmental venous anomalies are considered developmental variations in the venous drainage pathway for a portion of the brain parenchyma. ${ }^{1-4}$ DVAs are generally discovered incidentally and are considered benign. While there have been some reports of DVAs presenting with hemorrhage or neurologic symptoms, ${ }^{4-7}$ it is unclear and controversial whether this associ-

Received November 12, 2012; accepted December 5.

From the Mallinckrodt Institute of Radiology (A.S., G.J.Z., C.H., C.P.D.) and Departments of Neurology and Neurologic Surgery (G.J.Z., C.P.D.), Washington University School of Medicine, St. Louis, Missouri; Department of Radiology (A.S., G.J.Z., C.P.D.), Barnes-Jewish Hospital South, St. Louis, Missouri; and Department of Radiology (A.S., G.J.Z., C.P.D.), Saint Louis Children's Hospital, St. Louis, Missouri.

This work was supported by the Foundation for Barnes-Jewish Hospital.

Please address correspondence to Aseem Sharma, MD, Mallinckrodt Institute of Radiology, Washington University School of Medicine, Campus Box 8131, 510 S

Kingshighway Blvd, St. Louis, MO 63110; e-mail: sharmaa@mir.wustl.edu

http://dx.doi.org/10.3174/ajnr.A3516 ation reflects a causal relationship. There is a clear association between DVAs and cavernous malformations, and perhaps hemorrhage, with cavernous malformations found in up to $33 \%-48 \%$ of DVAs in some series. ${ }^{3,8}$

The reasons behind the tendency of some DVAs to be associated with symptoms or to develop CMs are not fully understood. Various mechanisms have been proposed for clinical manifestations of DVAs, including mechanical compression of the adjacent brain parenchyma, diminished venous outflow across the brain parenchyma drained by the DVA, increased flow related to arterialization of the DVA, stenosis of the draining vein, or the tortuosity of the venous tributaries. ${ }^{2,9}$ While $\geq 1$ of these factors may be at play in affecting risk of hemorrhage or CM formation in a given DVA, all these factors are likely to affect the hemodynamics of the blood flow in the brain parenchyma that the DVA is draining. In small case series of 3-4 cases, previous authors have shown that such altered hemodynamics may be manifest in the perfusion 
maps generated from the PWI. ${ }^{10,11}$ A more in-depth study into such perfusion abnormalities may help to better characterize focal hemodynamic alterations in the brain tissue drained by the DVAs and their potential association with hemorrhage/CM formation.

Our purpose was to study the perfusion alteration in the brain parenchyma drained by DVAs by using PWI and to investigate differences in the perfusion parameters around DVAs with and without CMs.

\section{MATERIALS AND METHODS}

This prospective study was approved by our institutional review board and conducted in compliance with the Health Insurance Portability and Accountability Act. All patients signed written consent documents to participate.

\section{Patient Selection}

The inclusion criteria were the presence of a brain DVA (with or without a CM) diagnosed on prior MR imaging, in the absence of other structural abnormalities of the brain. Potential candidates for the study were identified at our vascular-neurosurgery clinic and by searching the radiology report data base of our institution by using the terms "developmental venous anomaly," "DVA," "venous anomaly," and "venous angioma." The exclusion criteria were the following: 1) inability to clearly delineate the intraparenchymal course of the venous tributaries of the DVA; 2) midline location of the DVA that precluded a comparison with the contralateral normal brain tissue; 3) claustrophobia, contrast reaction, estimated glomerular filtration rate of $<60 \mathrm{~mL} / \mathrm{min}$, or any other contraindication to contrast-enhanced MR imaging; and 4) age younger than 18 years.

All qualifying patients were contacted via telephone and invited to participate in the study. A patient who agreed to participate underwent contrast-enhanced MR imaging that included PWI. We were able to identify 66 eligible patients. Of these, 24 (11 men and 13 women) opted to participate and underwent MR imaging between July 2009 and May 2011. The patients ranged from 21 to 75 years of age. At the end of the study, we had 15 DVAs without and 10 with CMs. Eighty-eight percent of DVAs (22/25) were supratentorial, most involving the frontal lobe $(6 / 25,24 \%)$. For DVAs without CMs, the presenting symptoms included headaches in 7 DVAs, and seizures in 1. Seven DVAs without CMs were discovered incidentally. For DVAs with CM, 4 were incidentally discovered, while the presenting symptoms included headaches in 4, seizures in 1 , and slurred speech in 1 . With the exception of 1 DVA with a CM in a patient who presented with slurred speech related to acute hemorrhage, it was unclear whether any of the symptoms were directly related to DVAs.

\section{Image Acquisition and Postprocessing}

MR imaging was performed on a 1.5T scanner (Avanto; Siemens, Erlangen, Germany). The scan included axial T1WI, axial FLAIR imaging, axial SWI, PWI, and postcontrast axial and coronal T1WI. In addition, MR venograms were obtained by using a time-of-flight technique. PWI was performed by using a dynamic susceptibility-weighted imaging technique with intravenous injection of $0.1 \mathrm{mmol} / \mathrm{kg}$ of gadoversetamide (OptiMARK; TycoHealthcare/Mallinckrodt, St. Louis, Missouri) at a rate of $4 \mathrm{~mL} / \mathrm{s}$.
The perfusion data were postprocessed on a postprocessing workstation (Leonardo; Siemens) by one of the authors (A.S. with $>10$ years of experience in neuroradiology) to generate standard perfusion maps for relative $\mathrm{CBV}$, relative $\mathrm{CBF}$, and relative MTT. The right middle cerebral artery branches within the Sylvian fissure ipsilateral to the DVA were selected to define the arterial input function.

\section{Calculation of Relative Perfusion Values for Brain Parenchyma around the DVAs}

For each perfusion map, we selected 3 ROIs in the brain tissue around the DVA tributaries, using the conventional imaging sequences to exclude the venous tributaries or the CM (Fig 1). Each region of interest provided a numeric value (along with an SD, which was not used for analysis) representing the perfusion of the brain in that region (Fig 1C). A corresponding value was calculated by using an identically sized region of interest in a mirror image location in the contralateral brain (Fig 1D). The ratio of these 2 values provided a measure of brain perfusion around a DVA for the given region of interest, relative to the contralateral brain with normal venous drainage. The arithmetic mean of the ratios for the 3 ROIs was used to calculate $\mathrm{rCBV}$, $\mathrm{rCBF}$, and $\mathrm{rMTT}$ representing the relative perfusion of brain around the DVA.

\section{Calculation of Relative Perfusion Values for Brain Parenchyma with Normal Venous Drainage}

To assure validity of our methods, we also calculated control perfusion values for the brain with normal venous drainage (ipsilateral to the DVA), relative to the contralateral side (Fig 1D). For these, $1-\mathrm{cm}^{2}$ ROIs were drawn on the perfusion maps in the white matter of the frontal lobe, parietal lobe, occipital lobe, and cerebellar hemisphere, unless one of these regions could not be included due to the presence of a large DVA. The arithmetic mean of ratios thus generated was considered to represent control values of relative perfusion for brain parenchyma with normal venous drainage, designated as cCBV, cCBF, and cMTT.

\section{Statistical Analysis}

Perfusion parameters around DVAs (rCBV, rCBF, and rMTT) were compared with corresponding control values (cCBV, cCBF, and cMTT, respectively). In addition, $\mathrm{rCBV}$, rCBF, and rMTT for DVAs without CMs were compared with the corresponding values for DVAs with CMs. Normal curves were fitted to data distributions, and the normality of the distributions was tested with the Shapiro-Wilk $W$-test. Ninety-five percent confidence intervals ( $95 \%$ CIs) were calculated for normally distributed data, and medians and ranges were calculated for non-normally distributed data. Equality of variances was tested with the O'Brien, BrownForsythe, Levene, and Bartlett tests. Differences between independent group means for normally distributed data with equal variances were tested with the Student $t$ test. For non-normally distributed data with equal variances, the nonparametric Wilcoxon rank sum test was used. For data with normal distributions but nonequal variances, the $t$ test (assuming unequal variances) was used. For comparisons of paired group means, if the differences were normally distributed, the paired $t$ test was used; if the differences were non-normally distributed, the Wilcoxon rank 

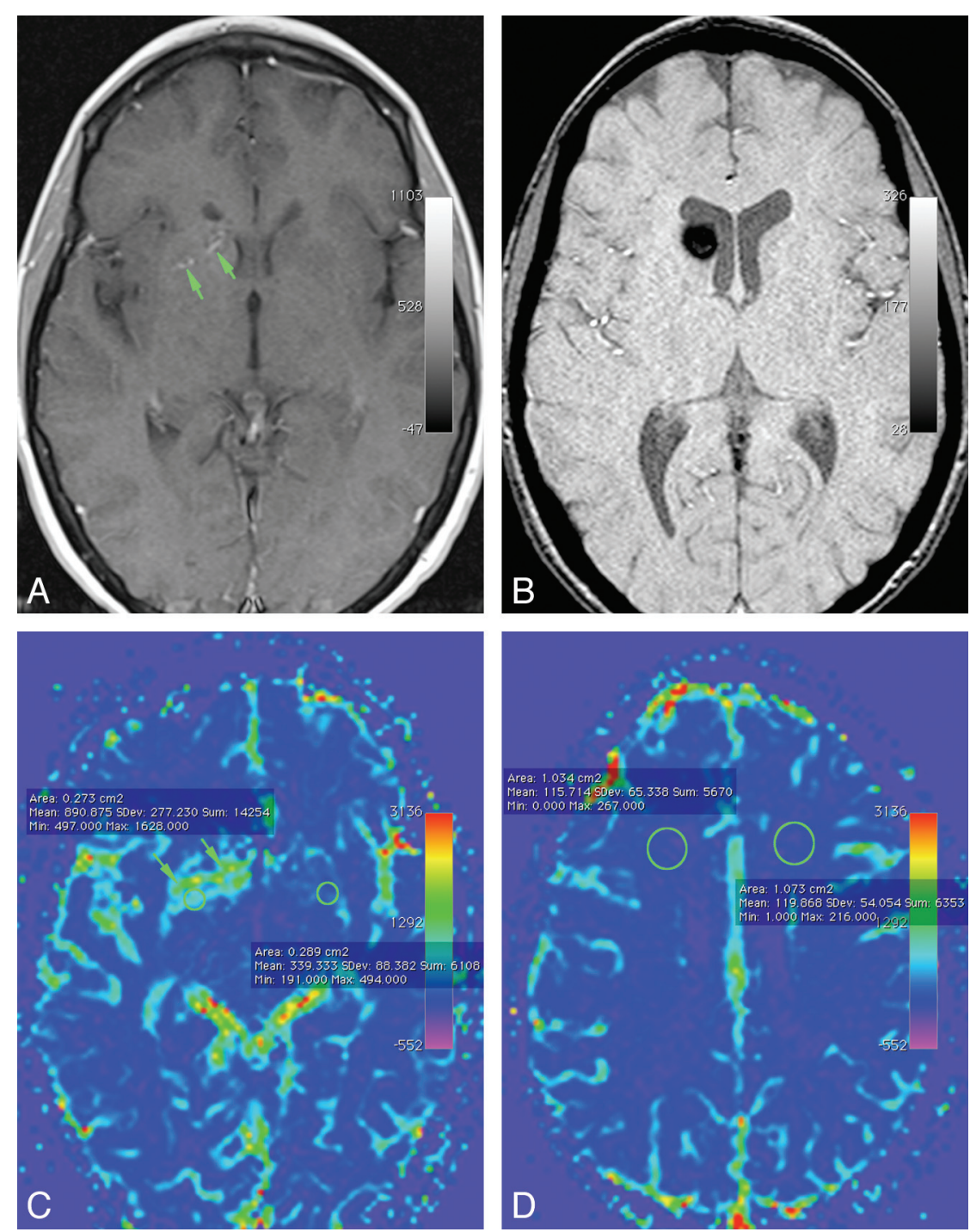

FIG 1. Axial contrast-enhanced T1-weighted $(A)$ and susceptibility-weighted $(B)$ images demonstrate a small developmental venous anomaly (arrows) in the right basal ganglia with a cavernous malformation in the right caudate head $(B)$. Corresponding cerebral blood volume map shows a wide zone of higher cerebral blood volume (indicated by green on this color map) in the brain around the DVA. Note that the elevation of CBV is not restricted to the location of the individual venous channels of the DVA (arrows) but involves a wider confluent zone of brain around the draining vein. Note placement of regions of interest to get the objective parameters for quantification of perfusion around DVAs $(C)$ and for brain with normal venous drainage $(D)$.

Table 1: Descriptive statistics for relative perfusion parameters for brain parenchyma drained by DVAs and for control regions with normal venous drainage

\begin{tabular}{lcccccc}
\hline Perfusion Parameter $^{\mathbf{a}}$ & Mean & $\mathbf{9 5 \%} \mathbf{C l}$ & Median & Minimum & Maximum & $\boldsymbol{P}_{\text {Value }}$ \\
\hline rCBV & 3.26 & $2.61-3.91$ & 2.98 & 1.39 & 6.61 & .04 \\
rCBF & 2.09 & $1.75-2.43$ & 2.00 & 0.79 & 4.43 & .19 \\
rMTT & 1.46 & $1.32-1.59$ & 1.44 & 1.00 & 2.14 & .15 \\
CCBV & 1.00 & $0.98-1.02$ & 0.99 & 0.89 & 1.06 & .15 \\
CCBF & 1.01 & $0.98-1.04$ & 1.00 & 0.94 & 1.27 & $<.01$ \\
CMTT & 1.00 & $0.98-1.02$ & 1.00 & 0.91 & 1.15 & .11 \\
\hline
\end{tabular}

a rCBV, rCBF, and rMTT represent relative cerebral blood volume, relative cerebral blood flow, and relative mean transit time respectively, for brain tissue around the DVA. CCBV, CCBF, and CMTT represent corresponding control values as measured in brain tissue with normal venous drainage (ipsilateral to the DVA)

${ }^{b} P$ value for Shapiro-Wilk $W$-test for data distribution normality. A value $<.05$ indicates a non-normal distribution

sum was used. For categoric data comparisons, the exact $P$ value for the Fisher exact test was calculated through data permutation. $\alpha$ was set at .05, and 2-tailed tests were used. Statis- tical analyses were performed with JMP Statistical Software, Release 9.0.0 (SAS Institute, Cary, North Carolina) and StatXact-9 statistical software for exact nonparametric inference (Cytel, Cambridge, Massachusetts).

\section{RESULTS}

\section{Perfusion Parameters}

For the perfusion parameters studied, Table 1 contains descriptive statistics and the results of testing data-distribution normality with the Shapiro-Wilk $W$-test. Normal-appearing brain surrounding the DVA showed increased rCBV (median = 2.98 , range $=1.39-6.61)$, increased $\mathrm{rCBF}$ (median $=2.00$, range $=0.79-4.43)$, and increased $\mathrm{rMTT}$ ( mean $=1.46$; $95 \%$ confidence interval, 1.32-1.59). The differences between rCBV and CCBV (median difference $=1.99$, range $=0.34-5.56)$ were non-normally distributed (ShapiroWilk $W$-test, $P=.04$ ), with rCBV being higher than CCBV (Wilcoxon signed rank test, $P<.01)$. The differences between $\mathrm{rCBF}$ and $\mathrm{cCBF}$ (mean difference $=1.08$; 95\% confidence interval, 0.75-1.41) were normally distributed (Shapiro-Wilk $W$-test, $P=.15$ ), with rCBF being higher than $\mathrm{CCBF}$ (paired $t$ test and Wilcoxon signed rank test, $P<.01$ ). The differences between rMTT and cMTT (mean difference $=0.46 ; 95 \%$ confidence interval, 0.32-0.59) were normally distributed (Shapiro-Wilk $W$-test, $P=.10$ ), with rMTT being higher (paired $t$ test and Wilcoxon signed rank test, $P<.01)$.

\section{Comparison of DVAs with and without CMs}

For the perfusion parameters in DVAs with and without CMs, Fig 2 contains plots of the values for perfusion parameters and Table 2 contains descriptive statistics and the results of testing data-distribution normality with the ShapiroWilk $W$-test. Data distributions for CBV in DVAs with and without CMs were normally distributed (Shapiro-Wilk $W$-test, $P \geq .16)$ and variances were equal (O'Brien, Brown-Forsythe, Levene, and Bartlett tests, $P \geq .36)$. The mean for rCBV in DVAs with CMs was 3.17 (95\% confidence interval, 2.01-4.34), and the mean for rCBV in DVAs without CM was 3.32 (95\% confidence interval, 2.45-4.20), with no difference between these mean values (Student $t$ test, $P=.82$ ). The means for cCBV for DVAs with 

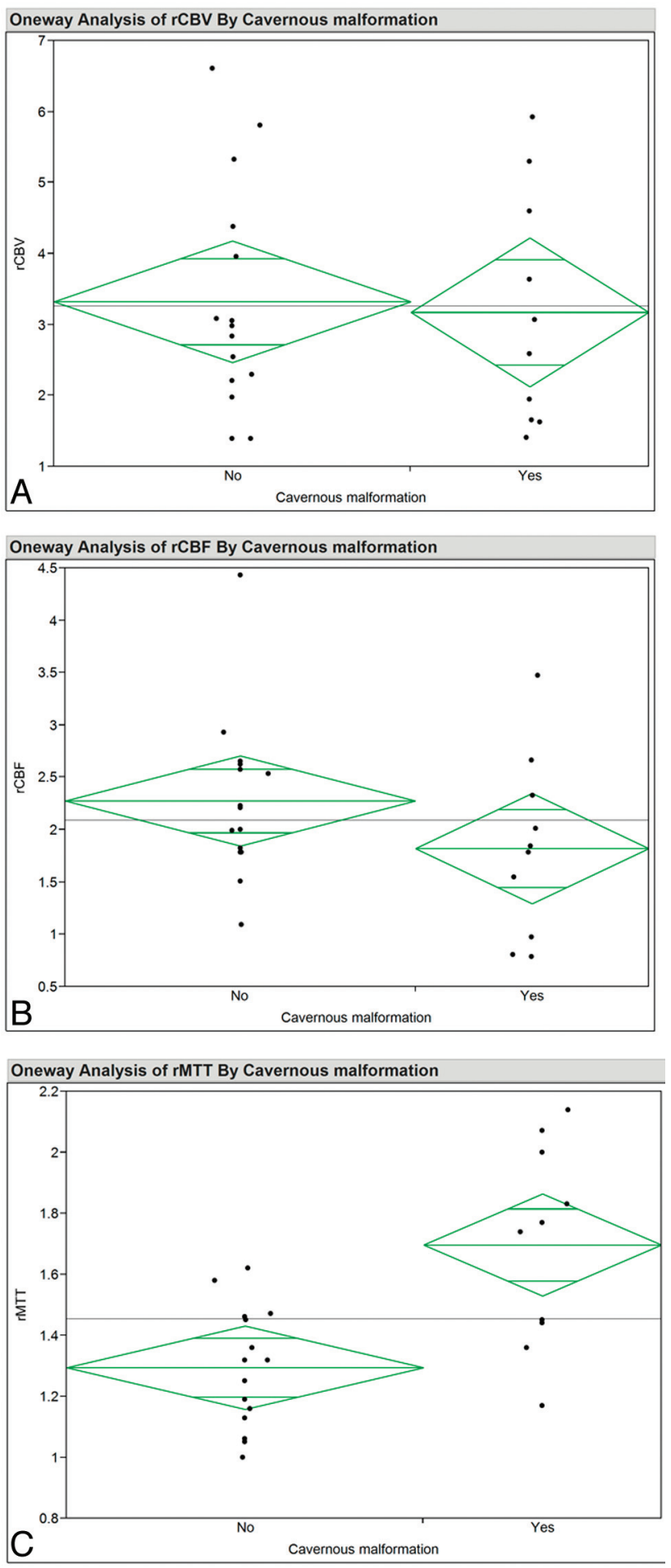

FIG 2. Ninety-five percent mean diamond plots for $r C B V(A), \operatorname{rCBF}(B)$, and $\mathrm{rMTT}(C)$ for patients with and without cavernous malformations. The horizontal line is the grand mean. The heights of the diamonds represent the $95 \%$ confidence intervals, and the widths of the diamonds are proportional to the sample sizes. If the overlap line of one diamond is closer to the mean of another diamond than is the overlap line of that diamond, there is no difference between the groups.

and without CMs were not significantly different (Student $t$ test, $P=.08)$.

Data distributions for CBF in DVAs with and without CMs were normally distributed (Shapiro-Wilk $W$-test, $P \geq .07$ ) except for cCBF in DVAs without CMs (Shapiro-Wilk $W$-test, $P<.01$ ). Variances were equal (O’Brien, Brown-Forsythe, Levene, and Bartlett tests, $P \geq .15)$. The mean for rCBF for DVAs with CMs was 1.82 (95\% confidence interval, 1.21-2.43), and the mean for rCBF in DVAs without CMs was 2.28 (95\% confidence interval, 1.85-2.70), with no difference between these mean values (Student $t$ test, $P=.18$ ). The means for $\mathrm{cCBF}$ in DVAs with and without $\mathrm{CMs}$ were not significantly different (Wilcoxon signed rank test, $P=.23$ ).

Data distributions for MTT in DVAs with and without CMs were normal (Shapiro-Wilk $W$-test, $P \geq .35$ ), and variances were equal (O'Brien, Brown-Forsythe, Levene, and Bartlett tests, $P \geq$ .36). The mean for rMTT in DVAs associated with a CM was 1.70 (95\% confidence interval, 1.46-1.93), and the mean for rMTT in DVAs not associated with a CM was 1.29 (95\% confidence interval, 1.19-1.40). Variances were not equal (O'Brien and Levene tests, $P \leq .04)$. The mean for rMTT in DVAs with a CM was higher than the mean for rMTT in DVAs without an associated CM $(t$ test assuming unequal variance, $P<.01$ ). The means for $\mathrm{cMTT}$ in DVAs with and without a CM were not significantly different (Student $t$ test, $P=.75$ ).

The location of DVAs did not differ significantly between the $\mathrm{CM}$ and non-CM groups (Fisher exact test, $P=.46$ ).

\section{DISCUSSION}

In view of the presence of some degree of altered perfusion around all the DVAs studied, our results indicate that the presence of a DVA is likely to be inherently associated with altered hemodynamics in the involved brain parenchyma. While supporting previous findings, ${ }^{10,11}$ our results indicate that PWI is able to detect such perfusion alterations around most DVAs, not just anecdotal cases. The pattern of perfusion abnormalities in our study was also similar to that seen previously, ${ }^{10,11}$ with higher relative CBV as well as MTT in the brain around the DVAs. A variable change in relative $\mathrm{CBF}$ (Fig 3) is also in accordance with that in previous studies. $^{10-12}$

The pathogenesis of DVAs is not fully understood. Some investigators consider them to result from in utero thromboses of

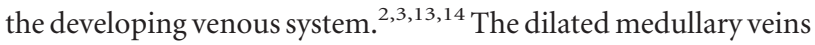
converging onto a prominent collector channel in effect serve as a replacement of the normal venous system that would have otherwise existed in this part of the brain. ${ }^{2}$ Consistent elevation of rMTT and rCBV seen in our study supports this model of DVA pathogenesis of a venous outflow, inherently less robust than normal venous drainage. Similar prolongation of MTT on PWI has been seen in the setting of other conditions affecting venous outflow, such as dural venous thrombosis or patients with SturgeWeber syndrome. ${ }^{15-17}$ This increase in the MTT for DVAs, however, appears to be due to alterations in the CBV and CBF that follow a pattern distinct from that seen in other restrictive venous pathologies. As per the central volume theorem, MTT is simply a ratio of $\mathrm{CBV}$ and $\mathrm{CBF} .{ }^{18}$ Prolongation of MTT in the presence of venous outflow restriction is usually the result of venous engorgement-induced increase in CBV, along with outflow restrictioninduced reduction in $\mathrm{CBF}$, as has been shown in diseases such as Sturge-Weber syndrome. ${ }^{16,17}$

In contrast, in most of the DVAs studied by us and in previous 
Table 2: Descriptive statistics for perfusion parameters in DVAs without and with CMs

\begin{tabular}{lccccccc}
\hline Perfusion Parameter $^{\mathbf{a}}$ & $\mathbf{C M}$ & Mean & $\mathbf{9 5 \%} \mathbf{C l}$ & Median & Minimum & Maximum & $\boldsymbol{P}$ Value $^{\mathbf{b}}$ \\
\hline rCBV & - & 3.32 & $2.45-4.20$ & 2.98 & 1.39 & 6.61 & .16 \\
& + & 3.17 & $2.01-4.34$ & 2.83 & 1.41 & 5.92 & .24 \\
rCBF & - & 2.28 & $1.85-2.70$ & 2.21 & 1.09 & 4.43 & .07 \\
& + & 1.82 & $1.20-2.43$ & 1.81 & 0.79 & 3.47 & .60 \\
rMTT & - & 1.29 & $1.19-1.40$ & 1.32 & 1.00 & 1.62 & .67 \\
& + & 1.70 & $1.46-1.93$ & 1.75 & 1.17 & 2.14 & .62 \\
$\mathrm{cCBV}$ & - & 1.01 & $0.99-1.03$ & 1.01 & 0.94 & 1.06 & .22 \\
& + & 0.98 & $0.95-1.02$ & 0.98 & 0.89 & 1.05 & .85 \\
$\mathrm{cCBF}$ & - & 1.02 & $0.98-1.06$ & 1.01 & 0.95 & 1.27 & $<.01$ \\
& + & 0.99 & $0.96-1.03$ & 0.98 & 0.94 & 1.08 & .33 \\
$\mathrm{CMTT}$ & - & 1.00 & $0.97-1.03$ & 1.00 & 0.91 & 1.15 & .35 \\
& + & 0.99 & $0.97-1.02$ & 0.99 & 0.94 & 1.05 & .99 \\
\hline
\end{tabular}

Note:- + , present; - , absent.

${ }^{a}$ rCBV, rCBF, and rMTT represent relative cerebral blood volume, relative cerebral blood flow, and relative mean transit time respectively, as measured in brain tissue around the DVA. CCBV, CCBF, and CMTT represent corresponding control values as measured in brain tissue with normal venous drainage (ipsilateral to the DVA).

${ }^{\mathrm{b}} P$ value for Shapiro-Wilk $W$-test for data distribution normality. A value $<.05$ indicates a non-normal distribution. in the venous development. Modest reactive vasodilation in arterioles has been demonstrated in response to venous dilation in animal studies. ${ }^{19}$ However, it is unlikely that such a reactive change is able to more than overcome the restrictive effects of the outflow restriction in the presence of venous outflow obstruction. The exact explanation for increased $\mathrm{rCBF}$ around DVAs remains unclear. However, it is possible that the developmental nature of the venous anomalies (as opposed to the acquired nature of venous obstruction in experimental models/pathologic venous obstruction) is somehow unique in that the etiologic factors responsible for formation of DVAs are also able to induce reports, the increase in rMTT was seen in the setting of increased rCBV and a less pronounced increase in the rCBF. While the increased $\mathrm{rCBV}$ is easy to explain, elevation of $\mathrm{rCBF}$ around many DVAs is more difficult to explain solely on the basis of alterations
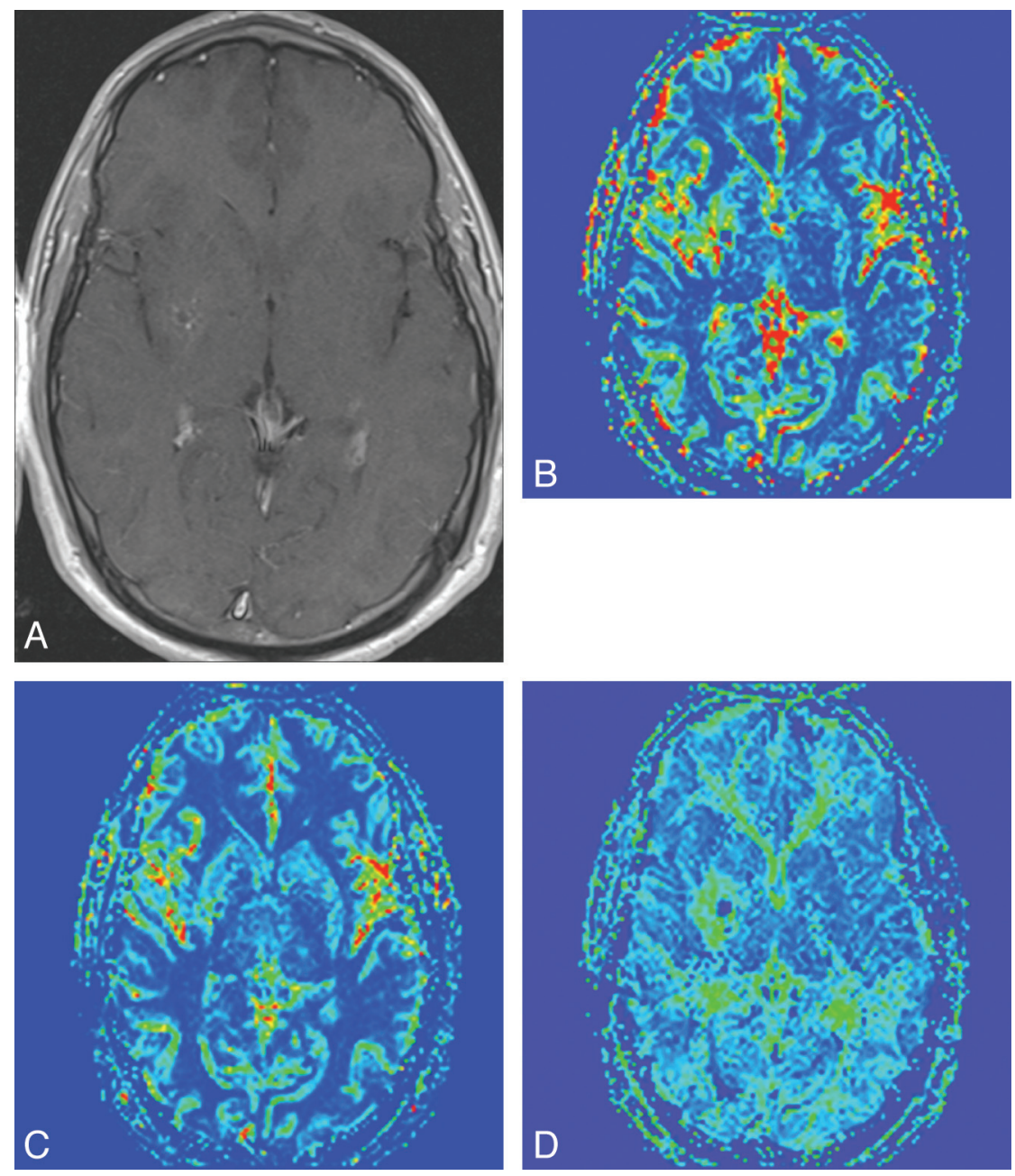

FIG 3. Axial contrast-enhanced T1-weighted image $(A)$ demonstrates tributaries of the DVA in the right lentiform nucleus, seen as punctate enhancing foci. Corresponding $r C B V(B), \operatorname{rCBF}(C)$, and $\mathrm{rMTT}(D)$ maps demonstrate a zone of perfusion alteration around these tributaries incorporating otherwise normal-appearing brain tissue. Note that in this case, the alteration in rCBV and rMTT maps was more pronounced relative to the rCBF. developmental changes in the arterial inflow that try to minimize stasis of blood in the affected brain by increasing the CBF in the face of increased CBV. A control cerebral blood flow indicator of close to 1 in brain parenchyma with normal venous drainage would argue against the possibility that these elevated relative CBF values are somehow representing artifacts.

Pathophysiologic implications of these results are also highlighted by the observed differences in the perfusion parameters of DVAs with and without CMs. A greater prolongation of MTT and a trend toward a lesser increase (or even decrease in individual cases) of $\mathrm{rCBF}$ in the $\mathrm{CM}$ group may be related to greater restrictive effects of DVAs in this group. Significant quantifiable differences in the perfusion parameters around developmental venous anomalies with and without associated cavernous malformations may reflect the role of hemodynamic factors in the development of these malformations. We hypothesize that in a large number of DVAs, the diminished venous capacity still has enough reserves to accommodate the physiologic needs, and such DVAs may remain completely asymptomatic. In other DVAs however, the restrictive effects on the venous drainage may be enough to cause local venous hypertension and thereby the propensity of these vessels to bleed and result in localized hemorrhage and subsequent CM formation. Given that the medullary veins composing the tributaries of the DVA are also known to have thinner walls, such veins may be more prone to bleed with this local venous hypertension. ${ }^{20}$

DVAs generally fill later in the venous phase on catheter angiography. Our find- 
ings of prolonged rMTT are consistent with this observation because it has been previously demonstrated that the angiographic circulation time is proportional to the mean transit time. ${ }^{21}$ Previously, authors have also reported some complex DVAs demonstrating early venous drainage, attributing it to microshunts within the DVAs or possibly concomitant DVAs and arteriovenous malformations. ${ }^{2,22,23}$ We did not have any patients with reduced rMTT as could be expected in these patients. While we did not have angiographic correlation for our patients, it is likely that given the rarity of such DVAs with microshunts, ${ }^{2}$ our study failed to include any such patients.

Our study had limitations. The number of patients was relatively small, and our results may not be generalizable to all DVAs. While PWI cannot accurately measure the actual CBV and CBF, it does offer a robust method to evaluate the relative changes in the perfusion of the brain. This is supported by the fact that for brain parenchyma with normal venous drainage, relative perfusion values (cCBV, cCBF, and cMTT) were noted to be close to 1 , as would be expected. It is possible that susceptibility effects of CMs can affect the relative perfusion values obtained in the surrounding brain parenchyma. While we cannot be certain of these additional susceptibility effects on the perfusion calculations, none of the ROIs were placed directly in the location of the CMs. In a similar manner, we took precautions not to place the ROIs directly on visible enhancing venous structures. Indeed, the correlation of the perfusions maps and the contrast-enhanced TIWI showed the perfusion alterations not to be restricted to the venous channels themselves but rather extending into the brain parenchyma in between the venous tributaries as well (Figs 1 and 3). However, it is possible that the elevated blood pool in the surrounding brain parenchyma could itself make the quantification of the perfusion parameters in this region less reliable. In a large number of individuals studied, the DVAs were either completely asymptomatic or the patient had symptoms that could not be directly ascribed to them. Similarly, acute hemorrhage was seen in only 1 DVA with an associated CM. It remains unknown whether DVAs with more pronounced symptoms or a greater propensity to bleed are associated with even greater hemodynamic perturbation.

CMs associated with DVAs are sometimes resected surgically. The decision to intervene, however, involves careful assessment of potential benefits and risks, including the risk of future expansion or hemorrhage of the CM. If the extent of altered hemodynamics in the surrounding brain tissue is associated with CM formation as indicated by our study, quantitative evaluation of such altered hemodynamics by using PWI may also provide a means for better assessment of future growth/hemorrhage of the CM. Future longitudinal studies will be needed to test this hypothesis.

Disclosures: Aseem Sharma—RELATED: Grant: Foundation for Barnes-Jewish Hospital.* Gregory Zipfel—UNRELATED: Grants/Grants Pending: National Institutes of Health,* McDonnell Center for Systems Neuroscience,* Washington University Institute of Clinical and Translational Sciences,* Washington University Center for Investigation of Membrane Excitability Diseases.* Colin DerdeynUNRELATED: Board Membership: WL Gore and Associates, Comments: medical device company developing products for ischemic stroke, Stock/Stock Options: nFocus, Pulse Therapeutics, Comments: medical device companies for brain aneurysms and stroke, respectively. ${ }^{*}$ Money paid to the institution.

\section{REFERENCES}

1. Jimenez JL, Lasjaunias $\mathrm{P}$, Terbrugge $\mathrm{K}$, et al. The trans-cerebral veins: normal and non-pathologic angiographic aspects. Surg $R a$ diol Anat 1989;11:63-72

2. Pereira VM, Geibprasert S, Krings T, et al. Pathomechanisms of symptomatic developmental venous anomalies. Stroke 2008;39:3201-15

3. Huber G, Piepgras U, Henkes H, et al. Venous anomalies of the brain: the clinical significance of the so-called venous angioma [in German]. Radiologe 1991;31:274-82

4. Lasjaunias P, Terbrugge K, Rodesch G, et al. True and false cerebral venous malformations: venous pseudo-angiomas and cavernous hemangiomas [in French]. Neurochirurgie 1989;35:132-39

5. Truwit CL. Venous angioma of the brain: history, significance, and imaging findings. AJR Am J Roentgenol 1992;159:1299-307

6. Campeau NG, Lane JI. De novo development of a lesion with the appearance of a cavernous malformation adjacent to an existing developmental venous anomaly. AJNR Am J Neuroradiol 2005;26: $156-59$

7. Garner TB, Del Curling O Jr, Kelly DL Jr, et al. The natural history of intracranial venous angiomas. J Neurosurg 1991;75:715-22

8. Ostertun B, Solymosi L. Magnetic resonance angiography of cerebral developmental venous anomalies: its role in differential diagnosis. Neuroradiology 1993;35:97-104

9. Hong YJ, Chung TS, Suh SH, et al. The angioarchitectural factors of the cerebral developmental venous anomaly; can they be the causes of concurrent sporadic cavernous malformation? Neuroradiology 2010;52:883-91

10. Camacho DL, Smith JK, Grimme JD, et al. Atypical MR imaging perfusion in developmental venous anomalies. AJNR Am J Neuroradiol 2004;25:1549-52

11. Kroll H, Soares BP, Saloner D, et al. Perfusion-CT of developmental venous anomalies: typical and atypical hemodynamic patterns. J Neuroradiol 2010;37:239-42

12. Matsuda $H$, Terada $T$, Katoh $M$, et al. Brain perfusion SPECT in a patient with a subtle venous angioma. Clin Nucl Med 1994;19: 785-88

13. Okudera T, Ohta T, Huang YP, et al. Developmental and radiological anatomy of the superficial cerebral convexity vessels in the human fetus. J Neuroradiol 1988;15:205-24

14. Saito Y, Kobayashi N. Cerebral venous angiomas: clinical evaluation and possible etiology. Radiology 1981;139:87-94

15. Doege CA, Tavakolian R, Kerskens CM, et al. Perfusion and diffusion magnetic resonance imaging in human cerebral venous thrombosis. J Neurol 2001;248:564-71

16. Lin DD, Barker PB, Hatfield LA, et al. Dynamic MR perfusion and proton MR spectroscopic imaging in Sturge-Weber syndrome: correlation with neurological symptoms. J Magn Reson Imaging 2006;24:274-81

17. Lin DD, Barker PB, Kraut MA, et al. Early characteristics of SturgeWeber syndrome shown by perfusion MR imaging and proton MR spectroscopic imaging. AJNR Am J Neuroradiol 2003;24:1912-15

18. Zierler $\mathrm{K}$. Indicator dilution methods for measuring blood flow, volume, and other properties of biological systems: a brief history and memoir. Ann Biomed Eng 2000;28:836-48

19. Wei EP, Kontos HA. Responses of cerebral arterioles to increased venous pressure. Am J Physiol 1982;243:H442-47

20. Abe M, Hagihara N, Tabuchi K, et al. Histologically classified venous angiomas of the brain: a controversy. Neurol Med Chir (Tokyo) 2003; 43:1-10, discussion 11

21. Gado MH, Phelps ME, Hoffman EJ, et al. Changes in cerebral blood volume and vascular mean transit time during induced cerebral seizures. Radiology 1976;121:105-09

22. Awad IA, Robinson JR Jr, Mohanty S, et al. Mixed vascular malformations of the brain: clinical and pathogenetic considerations. Neurosurgery 1993;33:179-88, discussion 188

23. Komiyama M, Mori $\mathrm{T}$, Nakajima $H$, et al. Cerebral venous angioma with parenchymal high intensity on $\mathrm{T} 2$-weighted images [in Japanese]. No To Shinkei 2001;53:88-89 